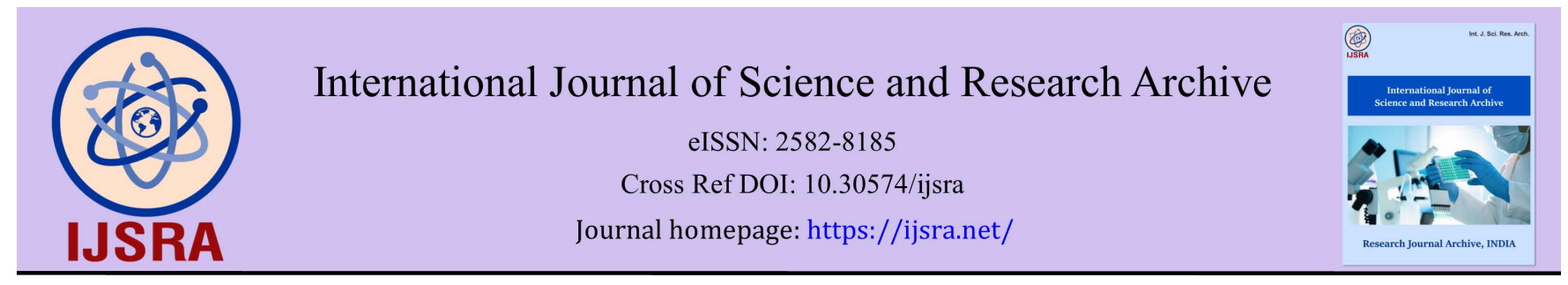

(RESEARCH ARTICLE)

\title{
Effects of substrates on the emergence and growth of Artemisia annua L. (Asteraceae) in soilless culture in Daloa (West Central Côte d'Ivoire)
}

\author{
Auguste-Denise Mambé BOYE, Elie Konan YOBOUET and Serge Kouadio N'GONIAN * \\ Laboratory for the Improvement of Agricultural Production; UFR Agroforestry, University Jean Lorougnon Guédé. BP150 \\ Daloa, Côte d'Ivoire.
}

International Journal of Science and Research Archive, 2021, 04(01), 198-209

Publication history: Received on 18 September 2021; revised on 24 October 2021; accepted on 26 October 2021

Article DOI: https://doi.org/10.30574/ijsra.2021.4.1.0165

\begin{abstract}
Artemisia annua is a plant used in traditional Chinese medicine to prevent and treat malaria and other parasitosis. However, the production of Artemisia annua seems to face many problems especially in the germination of grains making it difficult to grow this promising plant. The objective of this work is to contribute to the improvement of the emergence and growth of Artemisia annua in soilless culture. To do so, Artemisia annua seeds were sown in a germinator made of three organic substrates: simple soil, carbonized rice husk soil and soil-compost all supplemented with decomposed chicken droppings. After 24 days in the nursery, the seedlings were transplanted onto three growth substrates of the same composition as those in the nursery. The results showed that the substrate made of carbonized rice husk and soil, supplemented with chicken droppings is the best substrate. Plants growing on this substrate had a higher number of leaves (65 leaves/plant) with a height of $15 \mathrm{~cm}$ and a diameter of $9 \mathrm{~mm}$ than plants growing on the other two substrates. Moreover, the popularization of the results of this work will allow to intensify the culture of Artemisia annua but also to direct the actors of the sector of Artemisia annua towards new sustainable and more promising cultural techniques in order to eradicate malaria in Côte d'Ivoire and even in the sub-region.
\end{abstract}

Keywords: Artemisia annua; Substrates; Emergence; Growth; Soilless culture; Ivory Coast

\footnotetext{
${ }^{*}$ Corresponding author: Serge Kouadio N'gonian

Laboratory for the Improvement of Agricultural Production; UFR Agroforestry, Université Jean Lorougnon Guédé. BP150 Daloa, Côte d'Ivoire.
} 


\section{Graphical abstract}

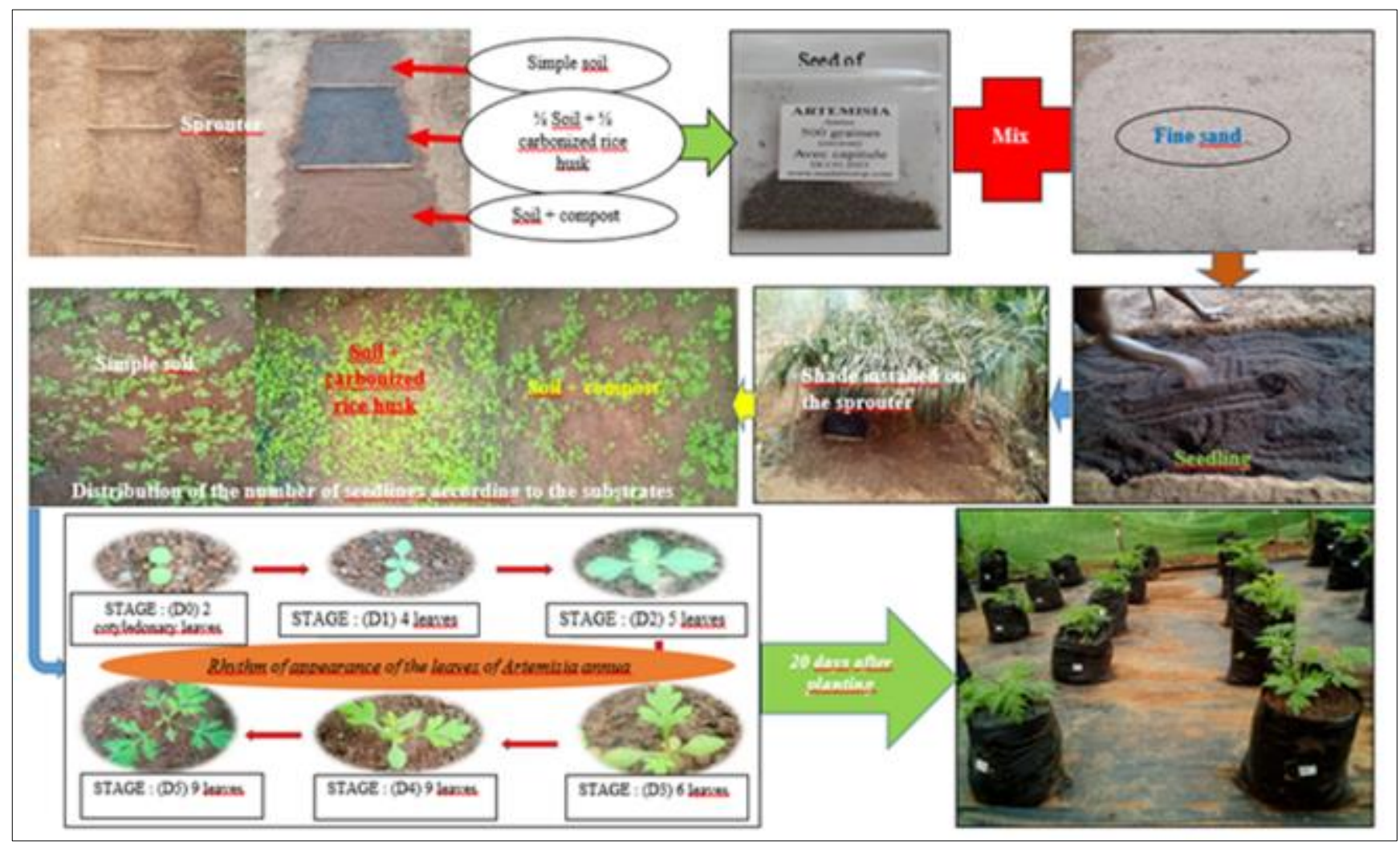

\section{Introduction}

The annual mugwort (Artemisia annua L.) is a species of the Asteraceae family native to the highlands of China. For about thirty years, it has been introduced in East Africa and Madagascar, then in West Africa (Burkina, Ivory Coast, Gambia, Mali, Nigeria, Senegal, Togo) and in South America (Brazil, Peru, Colombia) in a more marginal way [1; 2]. It is a herbaceous plant which develops preferably in not too heavy soils (sandy) with pH between 5.5 and 7.5 but also in soils with pH lower than 5.5 [2]. It has been used for 2000 years in Traditional Chinese Medicine to prevent and treat intermittent fevers (malaria) and other parasitosis [3]. Since its advent, studies tend to prove that the consumption of the whole plant in the form of herbal teas or capsules, would be just as effective against malaria as drugs containing artemisinin [4-5-6-7]. This traditional consumption constitutes a great hope for the populations to have access to a curative antimalarial treatment that is both effective and cheap. In addition, Artemisia annua is used to treat coccidiosis in hens and parasitemia (strongyles, strongyloides, coccidia and cestodes) in sheep [8].

However, according to Ferreira, many factors seem to be determinant in the production of Artemisia annua and must be refined for each geographical area [9]. Thus, in order to meet this challenge but also to enhance the value of this plant, a study was conducted in Daloa, West Central Côte d'Ivoire. The objective of this work is to improve the production of Artemisia annua in soilless on biological substrates. Specifically, it is to evaluate the effect of substrates on the emergence and growth of plants and to determine the ideal substrate for optimal growth.

\section{Material and methods}

\subsection{Plant material}

The plant material used was Artemisia annua seeds (Figure 1) provided by the Laboratory of Improvement of Agricultural Production of the University Jean Lorougnon Guédé Daloa.

\subsection{Technical equipment}

The tools used in this study are: a daba, a machete, a tape measure, a caliper, a sieve, a plastic, a digital camera, a watering can, an electronic scale. 


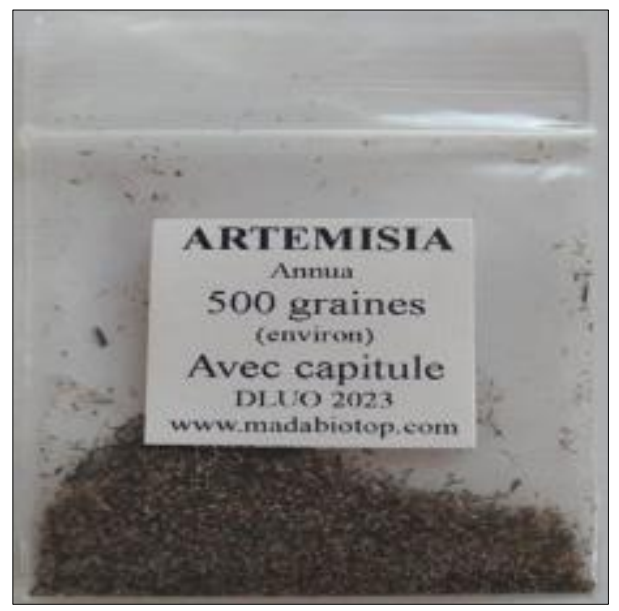

Figure 1 Seed of Artemisia annua L.

\subsection{Methods}

\subsubsection{Composting}

The compost used in the implemented experiment is based on crumbled fresh straw, chicken droppings and soil taken from the experimental site. The swathing is done on a flat surface. Water is added to achieve an optimal humidity of 50 to $60 \%$. The compost is turned once a week for one month.

\subsubsection{Carbonization of raw rice husks}

Carbonization is the transformation of an organic substance into charcoal, using heat (Figure 2). It is a very slow process, with a $120 \mathrm{~kg}$ bag of rice husks taking about 2.5 hours to be fully carbonized.

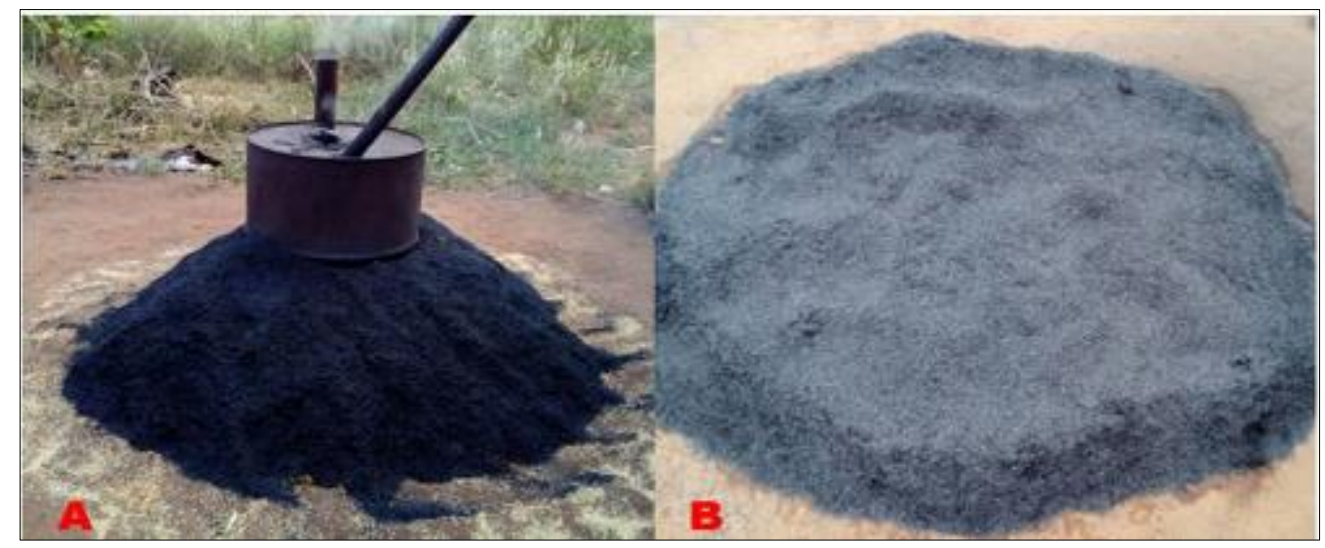

Figure 2 Carbonization of rice husks (A: Smothering of rice husks; B: Cooling)

The production of carbonized rice husks during this study was carried out in eight (08) steps described as follows:

Step ${ }^{\circ} 1$ : Make a carbonizer using a metal barrel with holes on the sides and two chimneys on the top;

Step $\mathrm{n}^{\circ} 2$ : Make a pile of dry wood with dry palms to the size of the barrel opening in the form of a cone on a flat, wellcleaned space;

Step $\mathrm{n}^{\circ} 3$ : Ignite the pile with a lighter or matchbox;

Step ${ }^{\circ} 4$ : Place the barrel on the wood pile 5 minutes after igniting;

Step $\mathrm{n}^{\circ} 5$ : Transfer the rice husk around the barrel 10 minutes later in order to form a pile in the shape of an inverted cone whose top will reach half the height of the barrel; 
Step $n^{\circ} 6$ : During the carbonization, it is necessary to stir the heap of husk to avoid that it enters in combustion until the whole takes a very dark color, almost black;

Step $\mathrm{n}^{\circ} 7$ : When the heap of bales has taken a very dark color (black), it is necessary to stop the carbonization by abundantly watering it with the jet so that it cools;

Step $n^{\circ} 8$ : Put the charred bale in a bag and store it in a dry place avoiding any contamination by harmful insects.

\subsubsection{Germination of Artemisia annua grains}

Preparation of the substrates

Before the constitution of the substrates, a trench of $15 \mathrm{~cm}$ x $180 \mathrm{~cm}$ x $50 \mathrm{~cm}$ was made using a daba, a pickaxe and a shovel. It was then divided into three equal compartments (Figure 3A). These were filled with the following substrates made from sieved soil, compost and carbonized rice husks (Figure 3B):

- T, a substrate made of sieved soil only;

- $\quad B$, a substrate made from a mixture of sieved soil (1/2) and carbonized rice husks (1/2);

- $\quad$ C, substrate consisting of sieved soil and compost (a $50 \mathrm{Cl}$ cup)

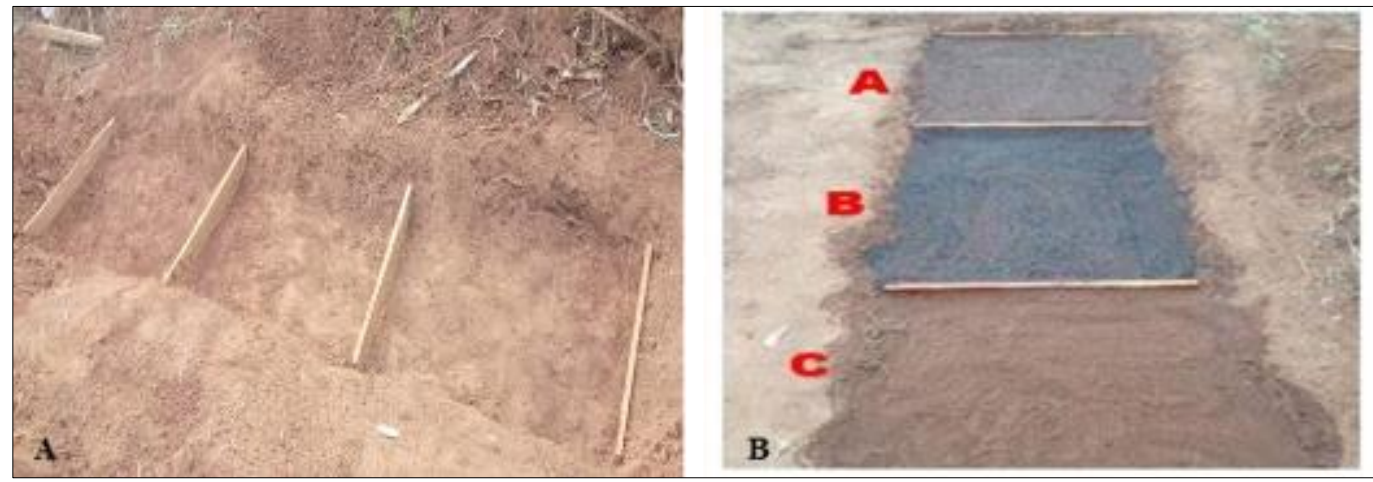

Figure 3 A: Compartmented trench; B: Trench filled with different types of substrates (A: Substrate T; B: Substrate B; C: Substrate C)

Sowing, maintenance and acclimatization
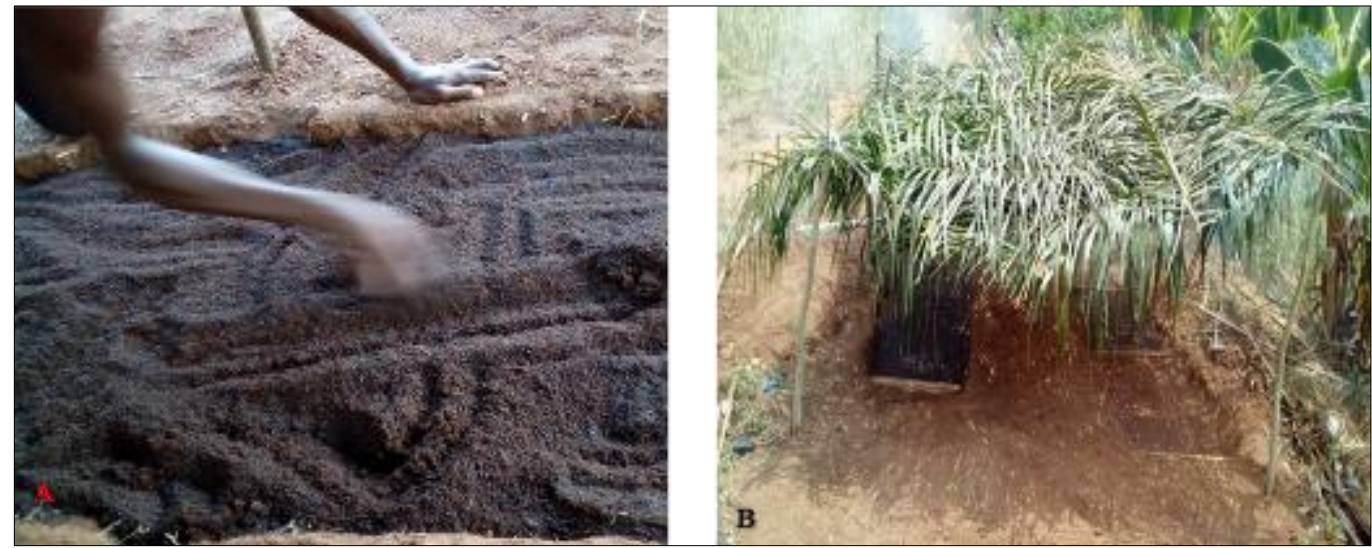

Figure 4 A: Sowing of Artemisia annua seeds; B: Shade installed over nursery substrates

Healthy (5 g) and mature Artemisia annua grains previously cleared of debris are mixed with fine river sand (50 g) in a tray. Then, they are spread at a rate of two handfuls of mature man per substrate (Figure 4A) followed by a sprinkling. 
The maintenance consisted of the construction of a shade over the nursery (Figure 4B), covering the seeded substrates with Artemisia annua seeds with a mosquito net and watering every two days. Twenty-one days after emergence, shading was gradually reduced until day 30 to allow the young seedlings to better adapt to the climatic conditions of the study area.

\subsubsection{Transplantation}

Site preparation

A plot of $27 \mathrm{~m}^{2}$ was cleared with a machete and then ploughed with a daba to obtain a bare and flat surface. A fence was built around the plot using mosquito nets to prevent any aggression to the plants. Plastic was then placed on the plot to isolate some of the emerging roots from the soil substrates.

Preparation of growth substrates and experimental set-up

The substrates previously prepared (during the nursery phase) were used to fill ninety (90) culture bags measuring 17 $\mathrm{cm} \times 24 \mathrm{~cm}$. The substrates were then arranged in double rows at a density of $50 \mathrm{~cm} \times 50 \mathrm{~cm}$. The device used is illustrated in Figure 5 below.

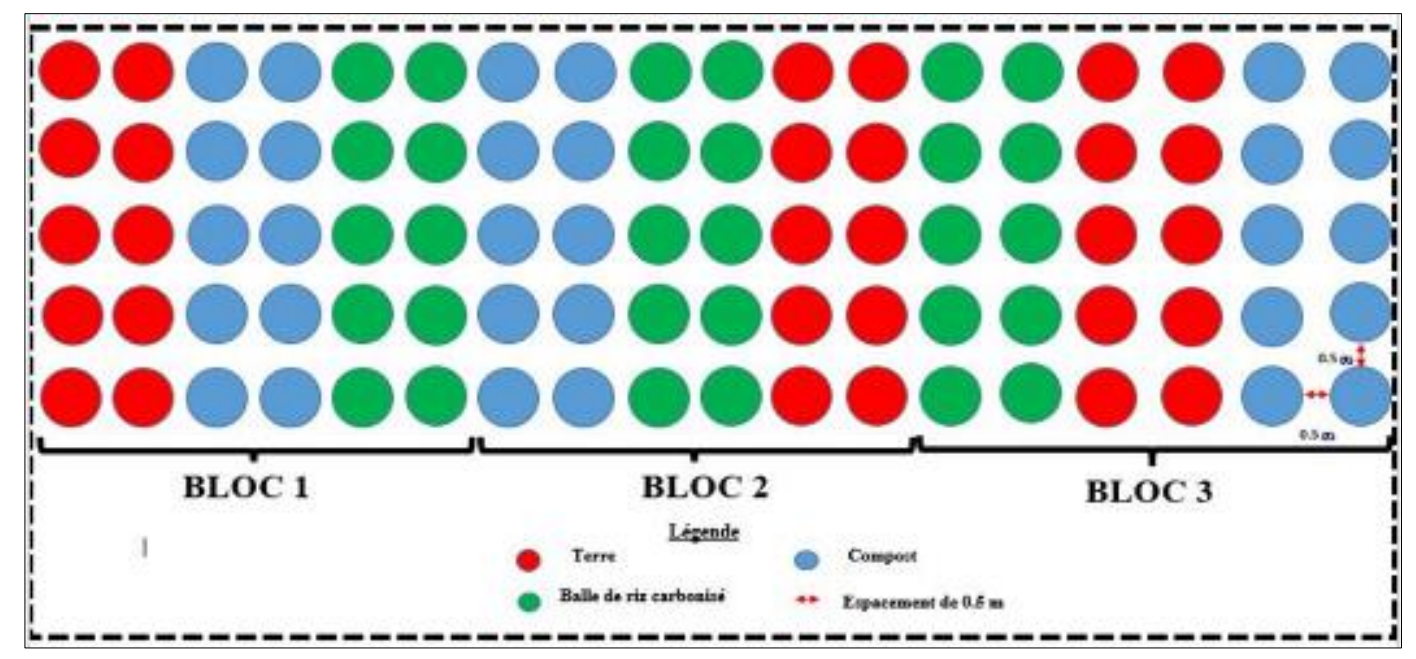

Figure 5 Experimental device

\section{Transplanting of the plants}

One hour before transplanting, the nursery was abundantly watered in order to facilitate the pulling out of the seedlings. Using a stake, an opening was made in the substrates according to the length of the seedling roots. Then, the vigorous seedlings aged 30 days with nine (09) true leaves were selected and transplanted to the collar on the culture substrates, taking care to accommodate the roots (Figure 6).
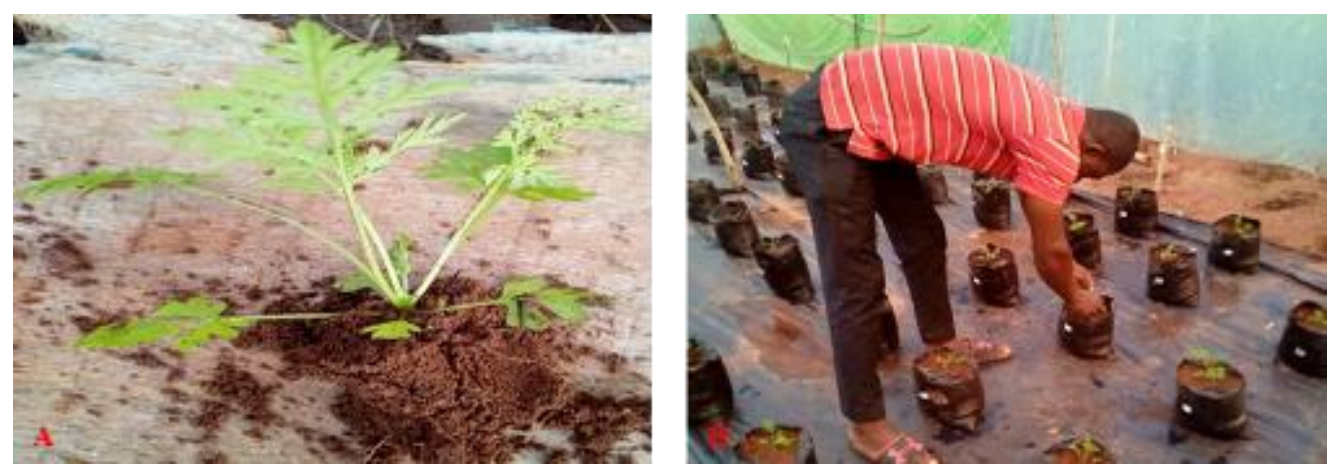

Figure 6 A: 30-day-old Artemisia annua plant; B: Transplanting of plants 


\section{Maintenance}

Maintenance consisted of weeding, replacing dead plants and watering. Indeed, in order to ensure the growth of the plants, the watering was limited to one watering every two days, preferably in the evening and to avoid watering after a strong rainfall. In addition, weeding consisted of removing all weeds that appeared on the surface of the substrates by hand. Dead plants were systematically replaced after observation.

\subsubsection{Data collection}

The observations made during this study were firstly the measurement of the $\mathrm{pH}$ and the temperature of the germination substrates with the help of a multifunctional pH meter. Secondly, the agro morphological parameters such as the height of the main stem, the diameter at the collar were measured respectively using a tape measure and a caliper. Then, the number of leaves and the number of branches were noted by counting.

\subsubsection{Statistical analysis}

The data collected on the different parameters were first entered using Excel 2016 software and then processed using XLSTAT 2019 software. Within each group, the substrates were compared to each other. A one-factor analysis of variance is performed to determine the effect of the different substrates on the studied parameters. In case of significant difference between the formulations, a post-hoc test is performed. This test allows to identify the parameter(s) that differ significantly according to the substrates.

\section{Results}

\subsection{Nursery phase}

\subsubsection{Number of seedlings}

This graph (Figure 7) shows the distribution of the number of Artemisia annua seedlings according to the substrates 4 weeks after sowing. The surface of the three germinators is identical and of $0.5696 \mathrm{~m}^{2}$.

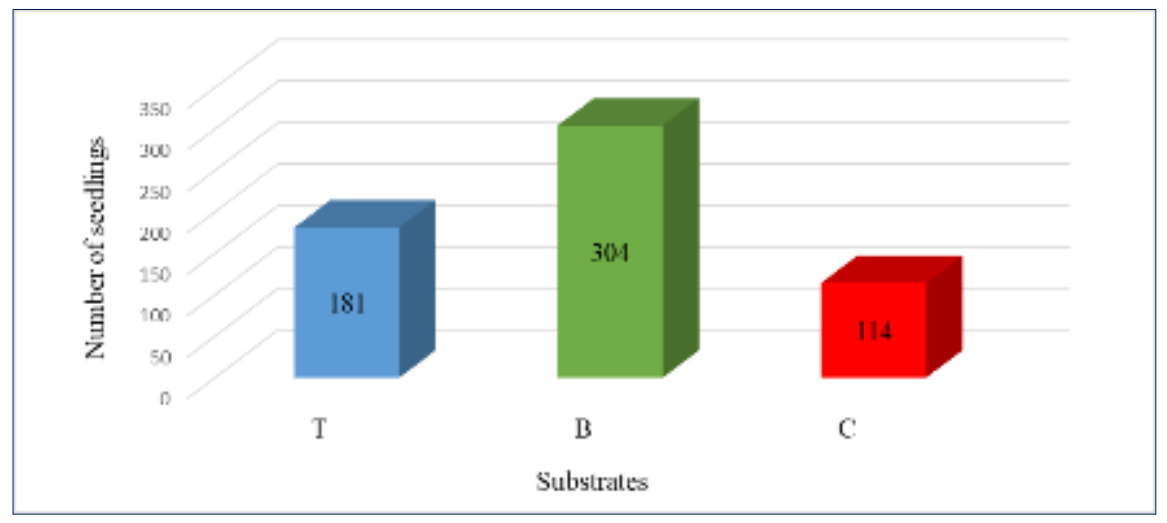

T: soil; C: soil containing compost; B: soil containing partially carbonized rice husk.

Figure 7 Distribution of the number of seedlings according to the substrates

The substrate containing partly carbonized rice husk (B) contains a higher number of seedlings, 304 seedlings observed against 181 and 114 respectively for Soil (T) and Compost (C).

\subsubsection{Average number of leaves and rate of leaf appearance}

The average number of leaves is the number of leaves per seedling for each substrate. Figure 8 shows the results of the average number of leaves per seedling as a function of the different substrates and time. Data were taken every 3 days. The best average number of leaves per seedling is observed in the soil substrate (T). The substrate made of soil has a high number of leaves per seedling, it is 09 leaves / seedling. The lowest numbers of leaves are observed in the substrate containing partly carbonized rice husk (B). The substrate containing compost (C) has seedlings with average numbers of leaves per seedling quite different but often much lower than 09 leaves/seedling. 
Figure 9 shows the different growth stages of Artemisia annua as a function of time. Six stages of growth are observed in the nursery, each corresponding to a foliage initiation by the plant. Thus, it is recorded that at each stage (within three (03) days), the young plant of Artemisia annua produces a true leaf except the two cotyledonary leaves until the 24th day after germination.

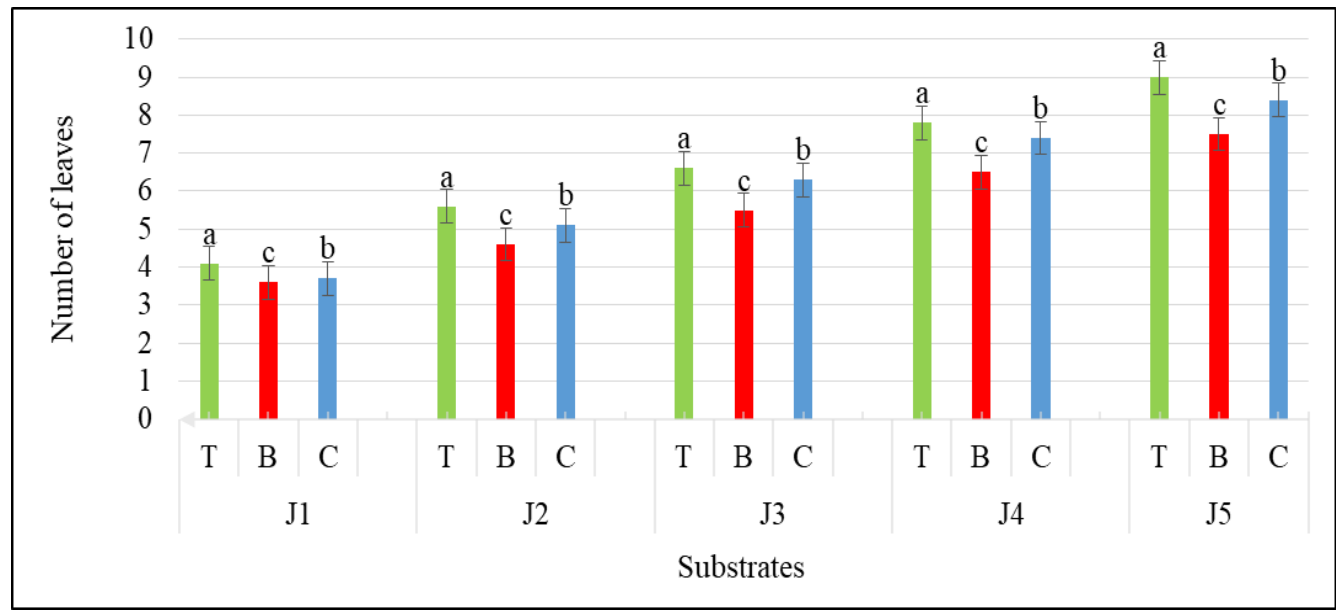

T: soil; C: soil containing compost; B: soil containing partially carbonized rice husk; D1: day 12; D2: day 15; D3: day 18; D4: day 21 and D5: day 24

Figure 8 Evolution of the number of leaves of seedlings as a function of time

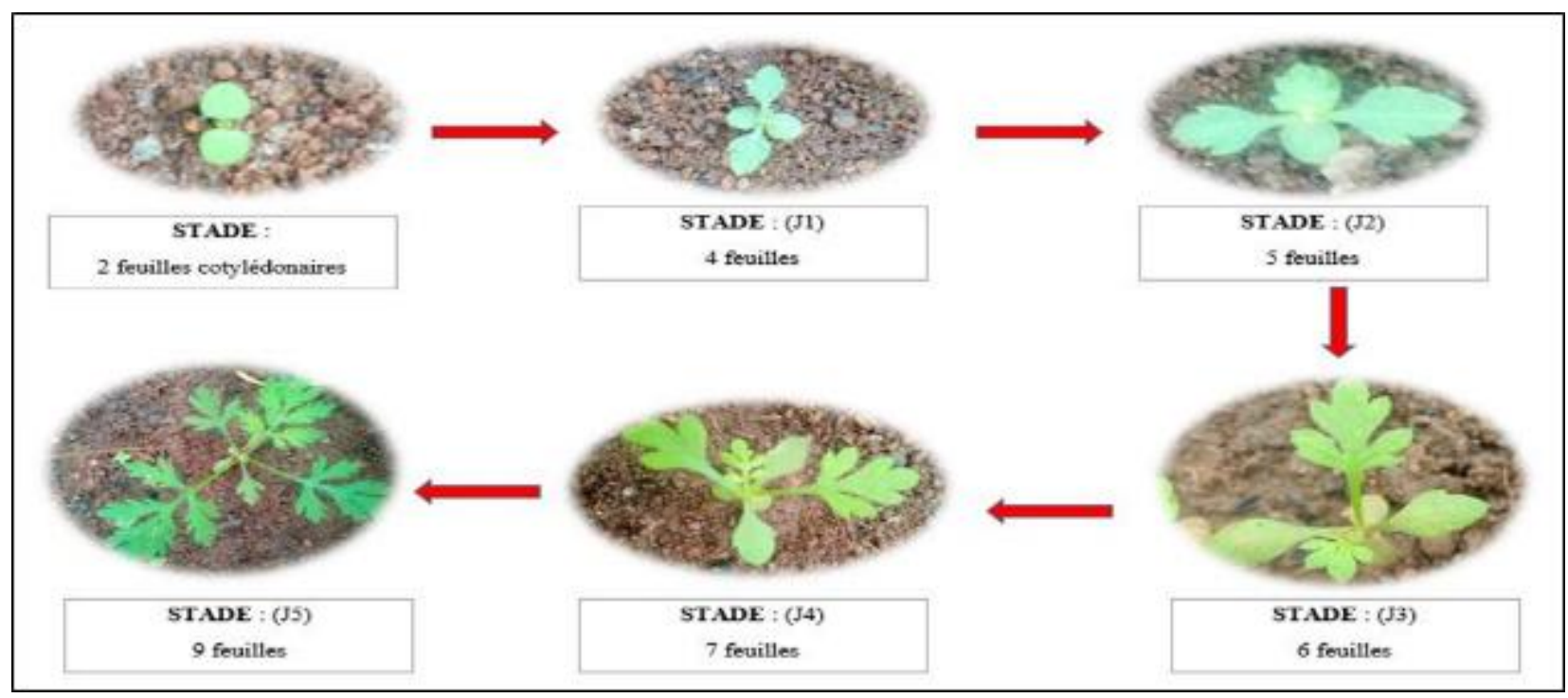

D1: day 12; D2: day 15; D3: day 18; D4: day 21 and D5: day 24

Figure 9 Different growth stages of Artemisia annua L.

\subsubsection{Temperature and $\mathrm{pH}$ of substrates}

The $\mathrm{pH}$ and temperature were taken at the end of the nursery phase (19 days). Table 1 shows the results for the temperature and $\mathrm{pH}$ parameters of three germination substrates. Analysis of the table shows that the $\mathrm{pH}$ ranged from 5.5 to 6.2. The $\mathrm{pH}$ results indicate that there is a significant difference between the prepared substrates. The carbonized rice husk substrate significantly increased the $\mathrm{pH}$ (5.5), while the soil-only and soil-compost substrates decreased the $\mathrm{pH}$ to 5.9 and 6.2, respectively. The results in Table 1 show that there is no significant difference between the temperatures of substrates $\mathrm{T}$ and $\mathrm{C}$ respectively $27^{\circ} \mathrm{C}$. However, the temperature of substrate $\mathrm{B}$ is significantly higher $\left(28^{\circ} \mathrm{C}\right)$ than that of the other two substrates. It appears from this analysis that all three substrates are acidic in nature; the carbonized ball soil substrate is more acidic with a high temperature than the other two. 
Table 1 Temperature and pH of the prepared substrates after 19 days

\begin{tabular}{|l|c|l|}
\hline Samples & Temperature $\left({ }^{\circ} \mathbf{C}\right)$ & $\mathbf{p H}$ \\
\hline $\mathrm{T}$ & $27 \mathrm{~b}$ & $5.9 \mathrm{~b}$ \\
\hline $\mathrm{B}$ & $28 \mathrm{a}$ & $5.5 \mathrm{c}$ \\
\hline $\mathrm{C}$ & $27 \mathrm{~b}$ & $6.2 \mathrm{a}$ \\
\hline
\end{tabular}

T: soil; C: soil containing compost; B: soil containing partially carbonized rice husk; TE: temperature; PH: pH of substrates

\subsection{Soil-less phase}

\subsubsection{Effect of substrates on the growth in height and diameter of plants}

Figures 10 and 11 show the results on the growth in height and diameter of Artemisia annua plants transplanted in three types of substrates. Plants growing in substrate B grew faster in height (15.08) and diameter (9.01) than those transplanted into substrates $\mathrm{T}$ and $\mathrm{C}$. The growth of these plants is followed by that of the plants in substrate T. On the other hand, the plants growing in substrate $\mathrm{C}$ have a slow growth in height and diameter. It is also observed that there is a significant difference between the different types of formulated substrates in height and diameter.

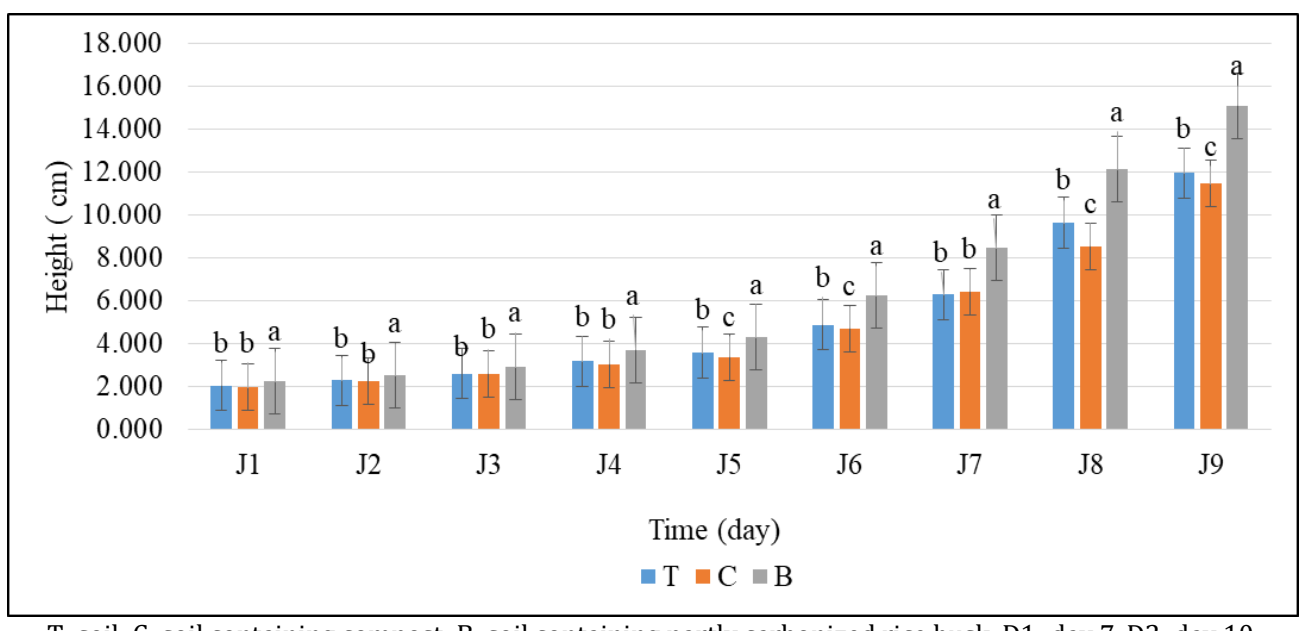

T: soil; C: soil containing compost; B: soil containing partly carbonized rice husk, D1: day 7, D2: day 10, D3: day 13, D4: day 16, D5: day 19, D6: day 22, D7: day 25, D8: day 28, D9: day 31

Figure 10 Height of the main stem of plants as a function of time

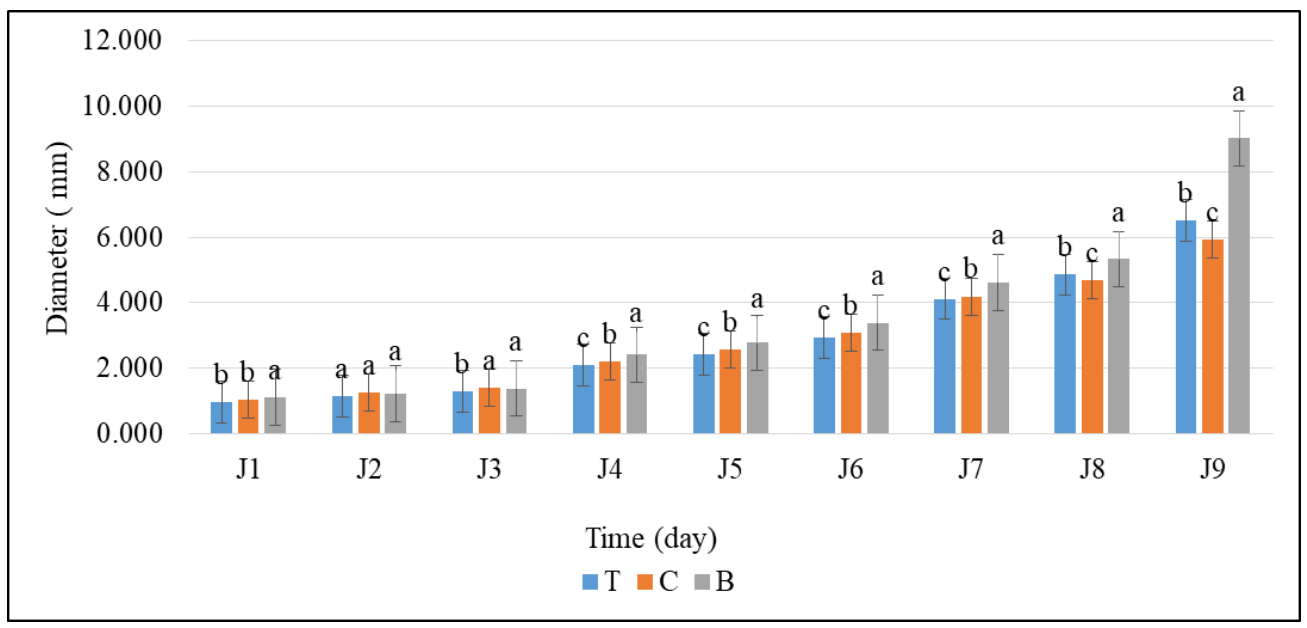

T: soil; C: soil containing compost; B: soil containing partially carbonized rice husk; D1: day 7; D2: day 10; D3: day 13; D4: day 16; D5: day 19; D6: day 22; D7: day 25; D8: day 28; D9: day 31.

Figure 11 Diameter of plants per substrate as a function of time 


\subsubsection{Effect of substrates on the number of shoots and leaves}

Figures 12 and 13 show the results for the number of shoots and leaves produced per plant according to the substrates. Analysis of this table reveals that plants grown on substrate B produced an average of 13 twigs and 65 leaves than those grown on the other substrates. Plants growing on substrate T produced a relatively higher number of shoots (11) and leaves (51) than those transplanted to substrate C (49 leaves and 11 shoots). However, there is a significant difference between the different substrates in the number of leaves. No significant difference is recorded between substrates $\mathrm{T}$ and $\mathrm{C}$ in the number of shoots.

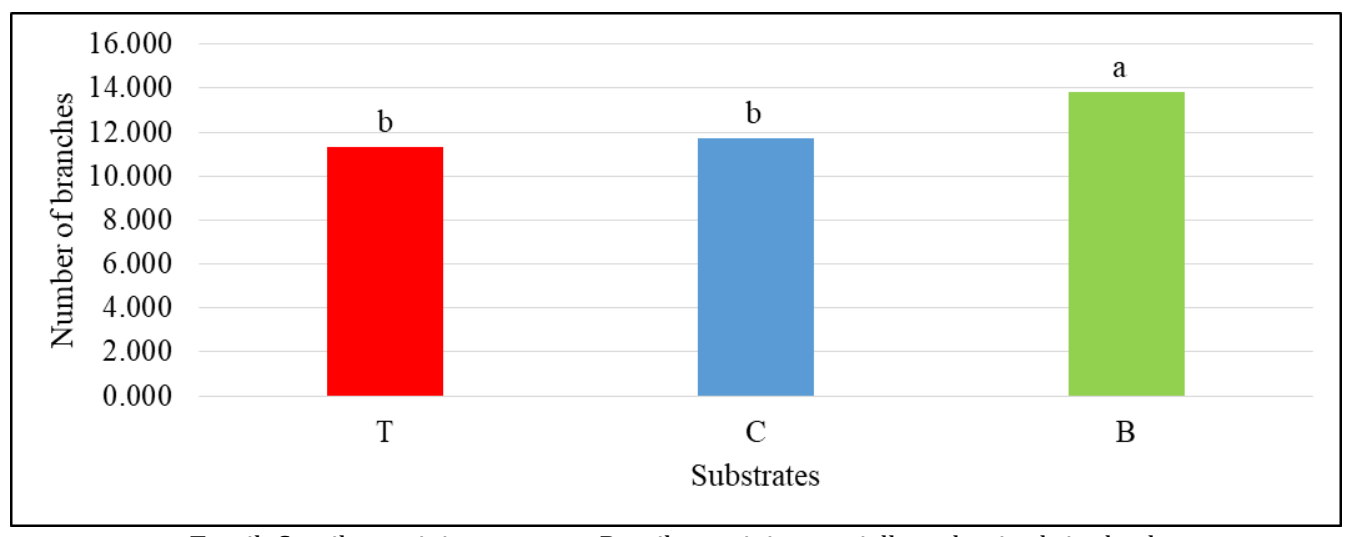

T: soil; C: soil containing compost; B: soil containing partially carbonized rice husk

Figure 12 Distribution of the number of plant shoots according to the substrates

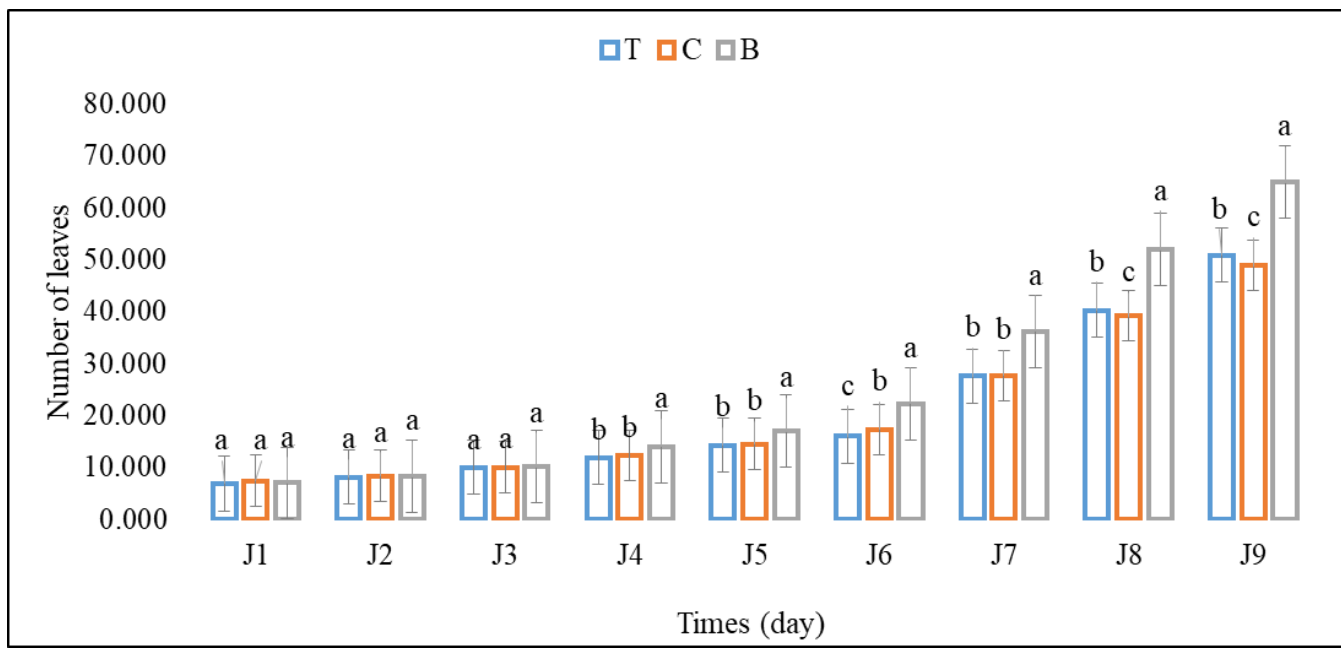

T: soil; C: soil containing compost; B: soil containing partially carbonized rice husk; D1: day 7; D2: day 10; D3: day 13; D4: day 16; D5: day 19; D6: day 22; D7: day 25; D8: day 28; D9: day 31.

Figure 13 Number of plant leaves per substrate as a function of time

\section{Correlation matrix (Pearson) of the variables}

The Pearson test indicates that there is a correlation between the different variables studied. The results of the analysis are presented in table 2 of the correlation matrix. From the results, it can be seen that there is a highly positive correlation between the height of the main stem and the number of twigs $(+0.850)$. There is also a strong positive correlation between the height of the main stem and the number of leaves $(+0.728)$ and on the other hand between the number of leaves and the number of twigs $(+0.689)$. There is also a weak positive correlation between the diameter at the collar and the three other variables, namely the height of the main stem $(+0.143)$, number of twigs $(+0.161)$ and number of leaves $(+0.064)$. 
Table 2 Correlation matrix (Pearson) for the variables

\begin{tabular}{|l|c|c|c|c|}
\hline Variables & Diameter at collar & Number of branches & Plant height & Number of leaves \\
\hline Diameter at collar & 1 & 0.161 & 0.143 & 0.064 \\
\hline Number of branches & & 1 & 0.850 & 0.689 \\
\hline Plant height & & & 1 & 0.728 \\
\hline Number of leaves & & & & 1 \\
\hline
\end{tabular}

\section{Discussion}

\subsection{Effect of substrates on seedling emergence}

The emergence parameters concern the number of seedlings per substrate, the number of leaves per seedling, the temperature and the $\mathrm{pH}$ of the substrates. Indeed, these parameters vary significantly according to the substrates studied. This is in line with the work of Touckia in his germination and growth trial at the juvenile stage of local strains of Jatropha curcas L. in Central African Republic [10]. They showed that germination parameters vary according to the substrates used.

Furthermore, the high number of seedlings on the carbonized rice husk substrate compared to other substrates could be explained by the germination tests of Lemieux on composted rice husks [11]. They observed an almost 50\% increase in the germination rate of tomato seeds on composted rice husks. Indeed, carbonization being also a technique of degradation of the natural substrate could promote, as composting, the increase of the germination rate observed on carbonized rice husks. The results of this study are consistent with those of N'Gonian in the evaluation of agronomic descriptors of two cassava varieties (Manihot esculenta Crantz) on ten substrates in Daloa [12]. They showed that the carbonized rice husk substrate promoted a very high regeneration rate of cassava minisprouts.

The results also revealed that the three substrates were acidic in nature with a pH between 5 and 7, which corresponds to ideal conditions for the growth of Artemisia annua. However, the substrate composed of carbonized rice husk soil showed higher acidity and temperature than the other two substrates. This corroborates the work of [2] in Artemisinin and its derivatives. Contributions of traditional Chinese medicine in the control of chemoresistant malaria and contemporary perspectives. He demonstrated that Artemisia annua is an herbaceous plant that grows best on soils with $\mathrm{pH}$ between 5.5 and 7.5. Our results are in agreement with those of M'Sadak in the evaluation of substrates and seedlings produced in forest nurseries [13]. These authors showed that despite the decrease in $\mathrm{pH}$ of the substrates, the nutrients were assimilable. In addition, the emergence test of Artemisia annua seedlings in three different types of germplasm showed that the minimum emergence time of the seedlings under the conditions of the study is six (06) days after sowing. This result differs from that obtained by [14] in Niger with an emergence time between 3 and 20 days. This result also differs from those of [15] in Campuac, France. He showed that the minimum emergence time of Artemisia annua grains is one month. In addition, the difference noted is partly explained by the substrate used.

\subsection{Effect of the substrates on the parameters of growth of plants in soilless soil}

The results obtained on the growth parameters of Artemisia annua plants showed that there is a significant effect of the substrate used on the diameter. This is in agreement with the results of the work of [16] on Garcinia kola which found a variation in growth parameters depending on the substrate. The growth of plants on carbonized rice husks is better than that obtained on simple soil and soil-compost substrates. According to Misra R in the composting methods at the farm level, rice husk has the ability to aerate the soil, to lighten it when it is too heavy and to provide a slight acidity which would promote better growth and development of Artemisia annua seedlings [17]. In addition, a similar observation was made by [18] who stated that the use of carbonized rice husks as a nursery substrate increases the number of roots, the number and length of new shoots and the number of leaves. Chicken droppings added two weeks after transplanting improved the physical, chemical and biological qualities of the substrates to which they were added. This is confirmed by Chabalier in the guide of organic fertilization in the meeting, which add that the poultry reject $70 \%$ of the nitrogen and phosphorus consumed in the excrement [19].

The results of the correlation matrix obtained show that there are significantly marked and positive correlations between all the parameters studied, $(\mathrm{P}=0.001<0.05)$. The number of leaves, plant height, collar diameter and number of branches of the plants vary in the same direction and are related to each other. This is consistent with observations 
made by [10] in the germination and growth trial at the juvenile stage of local strains of Jatropha curcas L. in the Central African Republic. They showed positive and high intensity correlations between the growth parameters of Jatropha curcas.

\section{Conclusion}

The study showed that in the germinator, the carbonized rice husk substrate with suitable $\mathrm{pH}$ and temperature reduced the emergence time of Artemisia annua grains from 14 to 25 days with a very high emergence rate. The results also showed that after transplanting, plants growing on the carbonized rice husk substrate grew faster than those growing on the simple soil and soil-compost substrates. Therefore, the carbonized rice husk substrate can be recommended for good germination and growth of Artemisia annua plants in soilless culture. However, further trials are needed to confirm the reduced development cycle of Artemisia annua. These include changing the agro ecological zone to compare the different results that will be collected and also biochemical analysis to determine the richness of active principle in the dry matter produced.

\section{Compliance with ethical standards}

\section{Acknowledgments}

We sincerely thank all the teacher-researchers who devoted their time to the scrutiny of this work.

\section{Disclosure of conflict of interest}

The authors declare that they have no competing interests.

\section{References}

[1] Patil GV, Dass SK, Chandra R. Artemisia afra and Modern Diseases. J Pharmacogenomics Pharmacoproteomics. 2011; $2: 105$.

[2] Alexander S. Artemisinin and its derivatives. Contributions of traditional Chinese medicine in the fight against chemoresistant malaria and contemporary perspectives. University of Lorraine, Faculty of Medicine of Nancy. 2008.

[3] The Artemisia House. http://maison-artemisia.org/ - 2020. Accessed on 29/09/2021.

[4] Willcox M, Gerard B, Genevieève B, Vikas D, Falquet J, Ferreira JFS, Graz B, Hans-Martin H, Hsu E, Damien P, Colin WW. Artemisia annua as a Tradisional Herbal Antimalarial. 2004; 43-59.

[5] Zime-Diawara H, Sissinto-Savi TY, Akogbeto OE, Ogouyemi-Hounto A, Gbaguidi FA, Kinde-Gazard D. Study of the efficacy and tolerance of a tea based on Artemisia annua L. (Asteraceae) grown in Benin for the management of uncomplicated malaria. Int. J. of Bi and Che. Sci. 2015; 9(2): 692-70.

[6] Elfawal MA, Towler MJ, Reich NG, Weathers PJ, Rich SM. Dried whole-plant Artemisia annua slows evolution of malaria drug resistance and overcomes resistance to artemisinin. Proceedings of the National Academy of Sciences of the United States of America. 2015; 112(3): 821.

[7] Daddy NB, Kalisya LM, Bagire PG, Watt RL, Towler MJ, Weathers PJ. Artemisia annua dried leaf tablets treated malaria resistant to ACT and i.v. articulate: Case reports. Phytomedicine International Jol of phytotherapy and phytopharmacology. 2017; 32: 37-40.

[8] Ahmadreza F, Keramat A, Seyed MR. Anticoccidial effects of Artemisia annua ethanolic extract: prevention, simultaneous challenge-medication, and treatment. 2017.

[9] Ferreira JFS. Nutrient deficiency in the production of artemisinin, dihydroartemisinic acid, and artemisinic acid in Artemisia annua L. J of Agri and F Che. 2007; 55(5): 1686-1694.

[10] Touckia G. I., Yongo O. D., Abotsi E. K., Francois W., \& Kouami K. Germination and juvenile growth test of local strains of Jatropha curcas L. in Central African Republic. European Scientific. 2015; 11.

[11] Lemieux G, Larochelle L. Report of a mission to Senegal from 5 to 15 December 1992; 35.

[12] N'Gonian KS, Boyé MA-D, Aka BJK. Evaluation of agronomic descriptors of two cassava (Manihot esculenta Crantz) varieties on ten innovative substrates in Daloa, Côte d'Ivoire. Wo Jol of Ad Re and Re. 2021; 11(03): 093-101. 
[13] M'Sadak Y, Elouaer MA, El Kamel R. Evaluation of substrates and seedlings produced in forest nursery. Boi et for des tro. 2012; 313 (3): 61-71.

[14] Soumaila M, Barmou S, Aboulaye A, Mahamane A, Kalid I, Boube M, Saley K. Germination and growth trial of Artemisia annua L. in Niger. Inter Jol of Bio and Che Scis. 2015; 9(1): 108-120.

[15] Barrié P. Artemisia annua or how to fight malaria. 2006.

[16] CNRA. National Center for Agronomic Research, Directorate of Innovations and Information Systems, Côte d'Ivoire. 2012.

[17] Misra RV, Hiraoka H, Roy RN. On-farm composting methods, FAO (Food and Agriculture Organization of the United Nations), Bangkok, Rome. 2005.

[18] Kikuno H, Muamba K, Shiwachi H, Micho O, Assiedu R, March. Mini tuber production of white yam (D. rotundata) using vines: Ja Jol of Tro Agri. 2006; 50(1): 1-3.

[19] Chabalier PF, Kerchove VV, Macary HS. Guide to organic fertilization in the meeting. Co-ed. CIRAD, Réunion. 2006.

\section{Author's short Biography}

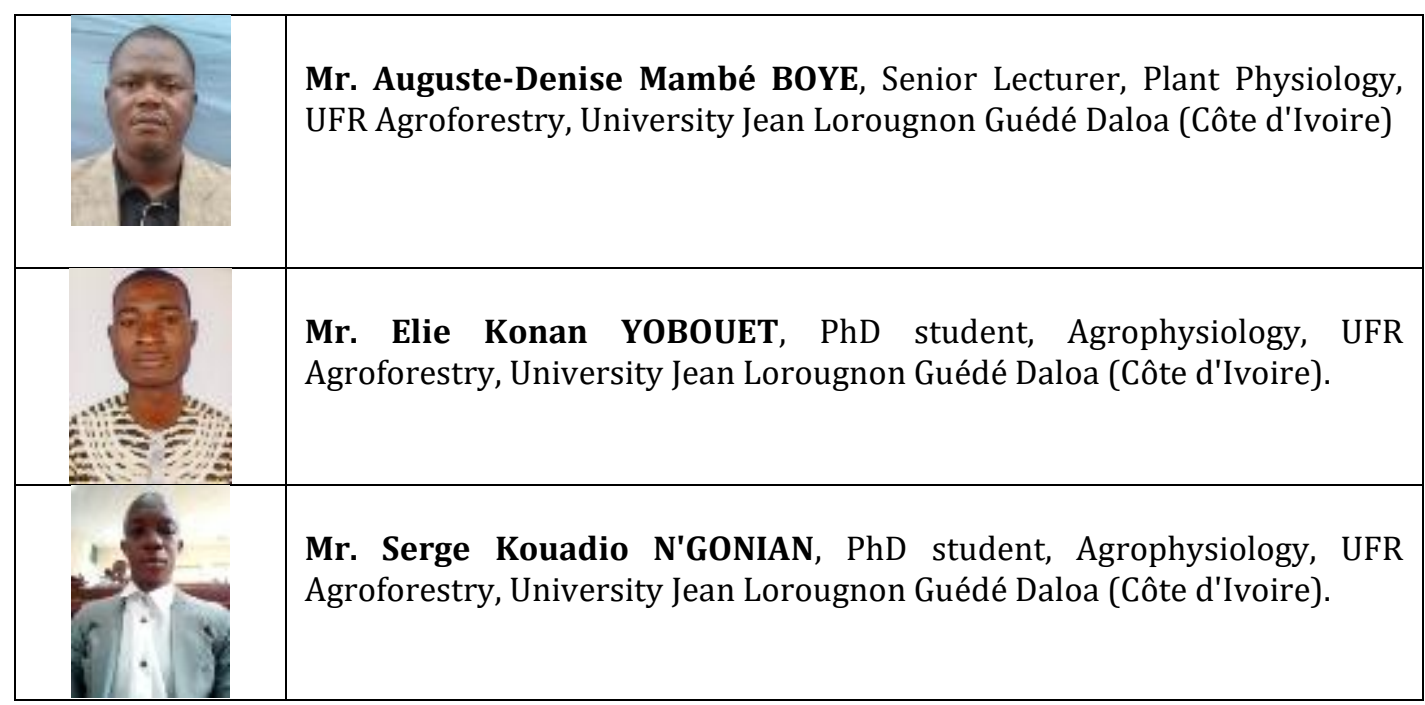

